\title{
RECEIVED
}

MAR 231998

OSTI CONF-971065--

\section{RECENT RESULTS FROM THE DIII-D TOKAMAK}

\author{
by \\ P.I. PETERSEN for the DIII-D TEAM
}

DISTRIBUTION OF THIS DOCUMENT IS UNLIMTED

FEBRUARY 1998 


\title{
RECENT RESULTS FROM THE DIII-D TOKAMAK
}

\author{
by \\ P.I. PETERSEN for the DIII-D TEAM
}

This is a preprint of a paper to be presented at the 17th IEEE/NPSS

Symposium on Fusion Engineering, October 6-11, 1997, San Diego, California and to be published in the Proceedings.

\author{
Work supported by \\ the U.S. Department of Energy \\ under Contract No. DE-AC03-89ER51114
}




\title{
Recent Results from the DIII-D Tokamak*
}

\author{
P.I. Petersen for the DIII-D Team ${ }^{\mathrm{a}}$ \\ General Atomics \\ P.O. Box 85608, San Diego, California 92186-9784
}

Abstract - The DIII-D national fusion research program focuses on establishing the scientific basis for optimization of the tokamak approach to fusion energy production. The symbiotic development of research, theory, and hardware continues to fuel the success of the DIII-D program. During the last year, a radiative divertor and a second cryopump were installed in the DIII-D vacuum vessel, an array of central and boundary diagnostics were added, and more sophisticated computer models were developed. These new tools have led to substantial progress in the understanding of the plasma. We now have a better understanding of the divertor as a means to manage the heat, particle, and impurity transport. Pumping of the plasma edge using the in situ divertor cryopumps effectively controls the plasma density. The evolution of diagnostics that probe the interior of the plasma, particularly the motional Stark effect diagnostic, has led to a better understanding of the core of the plasma. This understanding, together with tools to control the profiles, including electron cyclotron waves, pellet injection, and neutral beam injection, has allowed us to progress in making plasma configurations that give rise to both low energy transport and improved stability. Most significant here is the use of transport barriers to improve ion confinement to neoclassical values. Commissioning of the first high power $(890 \mathrm{~kW}) 110 \mathrm{GHz}$ gyrotron validates an important tool for managing the plasma current profile, key to maintaining the transport barriers. An upgraded plasma control system, "isoflux control," which exploits real time MHD equilibrium calculations to determine magnetic flux at specified locations within the tokamak vessel and provides the means for precisely controlling the plasma shape and, in conjuction with other heating and fueling systems, internal profiles. Disruptions are a design issue for ITER or any other future large tokamak. New disruption diagnostics and computer models have led to better understanding of disruptions, and tools such as impurity pellet injection have effectively reduced disruptive electro-magnetic forces and heat loads. Erosion from disruptions is found to contribute significantly to the erosion of tiles in the divertor region. The remote operation of DIII-D from other sites via the ESNET is providing better access for collaborators and helping develop such capability for ITER. Remote manipulation techniques have been used to repair the currently unused portion of the ohmic heating coil solenoid. This repair will increase the available ohmic heating flux swing by 2.5 Vs. Vanadium is being developed as a low activation material that can be used for the structure of future fusion devices. The DIII-D program plans to continue to develop theexperimental understanding and hardware systems to optimize the tokamak configuration both by improving the core performance and by developing the divertor configuration to manage the plasma edge.

\section{INTRODUCTION}

The mission of the DIII-D national program is to establish the scientific basis for the optimization of the tokamak approach to fusion energy production. The research, is carried out in four areas: transport, stability, boundary, and current drive and heating, is carried out in collaboration with a large number of national and international collaborators. The main goal of the research is to optimize the performance of the tokamak through active control of the plasma shape and the plasma profiles. In the transport area, the emphasis during the last year has been the study of the role of internal transport barriers in dramatically improving confinement. In the stability area, the emphasis has been on the study of neoclassical MHD, resistive wall stabilization, density limits, and disruption characterization and mitigation. In the boundary area the emphasis has been on developing understanding of radiative divertor physics and the initiation of the new radiative divertor, and in the current drive and heating area on the preparation of two $110 \mathrm{GHz}$ gyrotrons. These areas will be discussed in more details in the following sections.

The research program focuses on the DIII-D tokamak, which is a mid-sized device operating at near reactor level plasma parameters. It has a D-shaped cross-section with an aspect ratio of 2.5:1 and a major radius of $1.6 \mathrm{~m}$, and is capable of producing a large variety of plasma shapes, including elongation up to 2.6 and triangularity to 1.0 . The maximum toroidal field on axis is $2.1 \mathrm{~T}$, and the maximum plasma current is $3 \mathrm{MA}$. The shaping flexibility is due to the 18 fielding shaping coils that are located close to the plasma and individually controlled. A divertor baffle and a cryo pump are install above the floor and at the ceiling of the DIII-D vessel to allow study of divertor physics and control the plasma density. The auxiliary heating of the plasma is provided by eight neutral beams producing $20 \mathrm{MW}$ at $80 \mathrm{kV}$ in deuterium, $1.5 \mathrm{MW}$ (source) of $110 \mathrm{GHz}$ electron cyclotron heating, and $6 \mathrm{MW}$ (source) of fast wave current drive.

More than $\mathbf{5 0}$ different diagnostics are used to probe the DIII-D plasma. The Thomson scattering system measures the electron temperature and density every $2 \mathrm{~ms}$ at 40 different

\footnotetext{
*Work supported by U.S. Department of Energy under Contract No. DE-AC03-89ER51114.
} 
locations including the divertor region. The motional Stark effect diagnostic measures the local pitch angle of the magnetic field, and thereby of the plasma current density, and the radial electrical field. The charge exchange recombination diagnostic measures ion temperature, poloidal and toroidal rotation, impurity density, and radial electrical field. There are several diagnostics that measure fluctuations (beam emission spectroscopy, far infrared scattering, etc.). A digital control system, which control the plasma shape, current, and density and the injection of auxiliary heating power.

\section{SYSTEM UPGRADES}

To keep a research facility operating efficiently, it is necessary to continuously upgrade it as the understanding of the research topics is increased and new questions arise. The DIII-D vacuum vessel is normally opened once a year to make upgrades inside the vessel, and to clean, refurbish and calibrate existing components. Upgrades outside the vessel can be done during the year during the normal maintenance periods. Within the last year, a divertor baffle and an associated cryo pump were installed at the top of the DIII-D vessel. The baffle is similar to the one at the floor but with at a smaller major radius to create a more closed divertor that can pump high performance, high triangular plasma shapes. The pumping speed is similar to the lower pump, $40,000 \mathrm{l} / \mathrm{s}$ for deuterium. Halo current monitors were installed on the radiative divertor baffle plates and several halo current monitors were upgraded and relocated to better measure the poloidal and toroidal distribution of the halo currents. The monitors use normal carbon tiles that are electrically isolated from the vessel. A resistor is installed between the tile and the floor, and the voltage drop across the resistor is measured. Langmuir probes were installed on the radiative divertor plates and floor to measure the ion saturation current to and the electron temperature near these components. These data together with data from a number of other diagnostics (bolometer arrays and Thomson scattering) are used to validate the different divertor computer models. Several diagnostics, including the bolometer and soft x-ray arrays, were moved, since the new radiative divertor structure was blocking their views.

A second 16 channel view was added to the motional Stark effect (MSE) diagnostic. This diagnostic measures the pitch angle of the local magnetic field and the local electric field by measuring the polarization of the Stark split $D_{\alpha}$ lines from neutral particles injected by the neutral beam. The second view allows the magnetic field and the electric field components to be resolved. From this information, the qprofile and the radial electric field can be obtained.

We have installed two $110 \mathrm{GHz}$ gyrotrons operating at a nominal $1 \mathrm{MW}$ power level each. The first gyrotron was made by Gycom in Russia. It has an edge-cooled window of boron nitride, which limits the pulse length to $2 \mathrm{~s}$ at a power level of $1 \mathrm{MW}$. The second gyrotron is made by CPI (formerly Varian). It has a face-cooled sapphire window, which limits the power to $1 \mathrm{MW}$ for $0.8 \mathrm{~s}$ or $0.5 \mathrm{MW}$ for $2 \mathrm{~s}$. The gyrotrons have injected into the DIII-D through the transmission system, and the beam pattern and locations generated in the vacuum vessel correspond approximately to those calculated from the theory of Gaussian beam propagation and from ray tracing using 3D computer model. The resonance layer, which changes with the toroidal magnetic field, and the directional steering of the $\mathrm{ECH}$ wave is shown in Fig. 1.

A few years ago, DIII-D switched from an analog plasma control system to a digital system. The first step was a digital implementation of the analog control. Significant benefit resulted, since it was now possible rapidly to recall a previous control configuration. With this system the plasma crosssection was shaped by controlling a few parameters independently; these include the distance from the separatrix to the vessel ceiling, the distance from the inner wall to the separatrix and the location of the null point. During the last year a whole new control scheme has been developed. The new "isoflux" control [1] exploits the capability of a new real time EFIT algorithm to rapidly calculate magnetic flux at specified locations within the tokamak vessel. EFIT is an equilibrium code, which uses measurements that are obtained from diagnostics such as external magnetic probes, external poloidal flux loops, and the Motional Stark Effect (MSE) to calculate the plasma shape, and current and pressure profiles. In the new control system, the control parameters become the values of flux at pre-specified control point locations together with the $r$ and $z$ position of the separatrix $X$-point. Normally the control points are chosen to correspond to points on the separatrix flux surface of the EFIT-solution. In the old

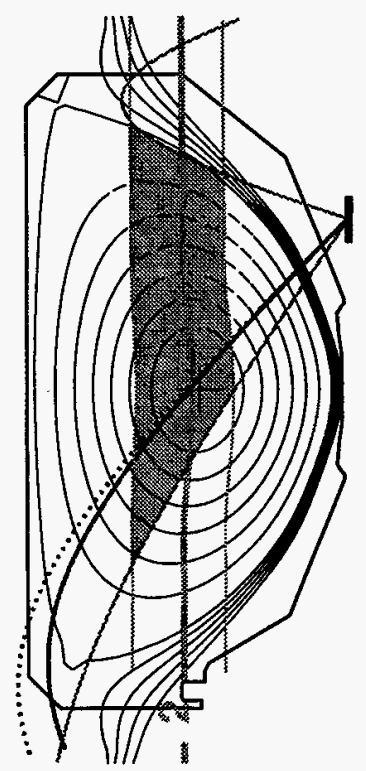

Fig. 1. Cross section of the DIII-D plasma with the ECH resonance layer and the path of the ECH wave. 
method, perturbations caused by one control parameter were ignored for the other parameter. This is not the case in the "isoflux control," where at least the linear response of the effect of perturbations of one parameter on other parameters is included.

The DIII-D ohmic heating coils was for practical reasons built with two solenoids in parallel. When a lead to one solenoid failed two years ago, after more than 15 years of service, we disconnected the damaged solenoid and ran with the other. The failed lead was not constructed correctly. All the other leads were inspected with borescope, and found to be properly constructed. The failed lead has now been successfully reinforced. Access to the lead area was gained by removing one of the ports in the floor of the DIII-D vessel to working at arms length to install a clamp assembly using a borescope to view the area. The clamping forces have been applied with remote sensors to indicate that the repair is functional. Two of three leaks in the cooling channel of the coil has been fixed, and work is in progress on the third. The ohmic heating coil circuit will then be returned to its original configuration to take advantage of the second solenoid and the extra $2.5 \mathrm{Vs}$.

As fusion devices get bigger and with increased collaboration, remote control of diagnostics and the device becomes more important. In order to demonstrate this capability, the DIII-D tokamak was remotely controlled from Lawrence Livermore National Laboratory (LLNL). The session leader, physics operator and neutral beam physics operator were located at LLNL, whereas the hardware operators where located at DIII-D. Audio and video communications were established between several people at the two sites. The plasma parameters were controlled from LLNL and all the plasma data were available for them to control the plasma and make decisions on how to progress in the experiment. The experiment was successful, and the distributed control had only minor impact on the execution of the experiment.

Vanadium-chromium-titanium alloys are attractive materials for fusion systems because of their strength, high temperature capability, and potential for low neutron activation and rapid activation decay. General Atomics, along with Argonne National Laboratory (ANL) and Oak Ridge National Laboratory, has developed a plan for utilization of vanadium alloys in the DIII-D tokamak which will culminate in the fabrication, installation, and operation of a vanadium alloy structure in the next divertor upgrade. The use of a vanadium alloy will provide a meaningful step towards developing advanced materials for fusion by 1 ) demonstrating the inservice behavior of a vanadium alloy ( $\mathrm{V}-4 \mathrm{Cr}-4 \mathrm{Ti})$ in a tokamak environment and 2) developing knowledge and experience on the design, processing, and fabrication of full scale vanadium alloy components. Small vanadium coupons have been, and are continuing to be exposed in DIII-D at positions in the vessel floor behind the lower divertor structure. They are then evaluated at ANL to determine the effects of the tokamak environment on the alloy.

\section{PHYSICS RESULTS AND NEW UNDERSTANDING}

Much has been learned about plasma heating and confinement in recent years, and understanding of plasma confinement and heating has been developed to a point that is sufficient for the design and construction of an experimental thermonuclear reactor [2]. However, new discoveries and understanding of improved confinement in advanced tokamaks might make it possible to design and build a smaller and more affordable reactor based on advanced tokamak concepts. An "Advanced Tokamak" [3] is a tokamak with enhanced confinement and normalized beta in near steady-state conditions obtained through active manipulation of the plasma profiles and cross sectional shape. In addition to the improvement in confinement, a steady-state tokamak requires: heat load and impurity control (divertor), disruption avoidance, and non-inductive current drive.

\section{A. Improved Confinement by Plasma Rotation}

Several confinement modes are observed in the DIII-D and other tokamaks. They are L-mode (Low), H-mode (High), VH-mode (Very High), NCS-mode (Negative Central Shear, also called RS for Reverse Shear), high $\ell_{\mathrm{i}}$ mode and high $\boldsymbol{\beta}_{\mathrm{p}}$ mode. L-mode [4] is obtained in an ohmic plasma or a plasma with low power auxiliary heating. $\mathrm{H}$-mode [5] was first discovered in a diverted plasma with neutral beam heating, but has since been obtained in other shapes and with other heating methods, and in other magnetic confinement devices: stellarator, heliotron/torsatron, and tandem mirror machine. The $\mathrm{H}$-mode confinement enhancement over $\mathrm{L}$-mode is a factor of 2-3. The $\mathrm{H}$-mode has a transport barrier (an area with low energy diffusivity) near the edge of the plasma. VH-mode [6] is a further confinement improvement over $\mathrm{H}$-mode with a broader transport barrier. In NCS mode [7], the transport barrier is located in the core of the plasma. Strong negative shear has produced the steepest ion temperature and toroidal rotation profiles seen yet in DIII-D [8].

One of the scientific successes of fusion research is the development of a model [9] to explain the formation of transport barriers in all the modes mentioned above. The fundamental physics involved in transport reduction is the effect of ExB velocity shear on the growth and radial extent of turbulent eddies in the plasma. The stabilization is due to two effects. The first is that the presence the $E \times B$ velocity shear results in enhanced damping by coupling the unstable modes to other, nearby, more stable modes. The second is reduction in radial transport owing to a decrease in the radial correlation length and the change in the phase between density, temperature, and potential fluctuations. The reason that the velocity shear plays a role in so many different 
situations is that there are a number of ways to establish the radial electric field $E_{\mathrm{r}}$. The radial force balance equation can be written as

$$
E_{r}=\left(Z_{i} e_{i}\right)^{-1} \nabla P_{i}-v_{\theta i} B_{\phi}+v_{\phi i} B_{\theta}
$$

where $Z_{i}$ is the ion charge number, e the electron charge, $n_{i}$ is the ion density, and $\nabla P_{i}$ is the gradient of the pressure; $v_{\phi i}$ and $v_{\theta i}$ are the toroidal and poloidal fluid velocities, and $B_{\phi}$ and $B_{\theta}$ are the toroidal and the poloidal magnetic fields. Thus, the electric field can be created by the pressure gradient, the poloidal flow and/or the toroidal flow. In DIII-D, where the neutral beams are injected in the direction of the plasma current, $v_{\phi} B_{\theta}$ is the major contributor to the radial electric field. Fig. 2 is shows a comparison of $\mathrm{L}$-mode and $\mathrm{H}$-mode discharges near the time of an $\mathrm{L}$ to $\mathrm{H}$ transition in a double null 1.5 MA plasma. The toroidal field is $2.2 \mathrm{~T}$, and $8.6 \mathrm{MW}$ of deuterium neutral beam power is injected into the plasma, which has a line average density of $3.6 \times 10^{19} \mathrm{~m}^{-3}$. The $\mathrm{L}$-mode time is $50 \mathrm{~ms}$ prior to the start of the dithering transition, while the $\mathrm{H}$-mode is $50 \mathrm{~ms}$ later in the quiescent $\mathrm{H}$-mode phase. In the $\mathrm{L}$-mode, the radial electric field $\mathrm{E}_{\mathrm{T}}$ changes gently from the center to the edge, and the $E \times B$ velocity shear rate $\omega_{\mathrm{E} \times \mathrm{B}}$ is comparable to the nonlinear turbulence decorrelation rate $\Delta \omega_{\mathrm{D}}$ in the whole plasma. The vertical bar indicates the uncertainty in $\omega_{\mathrm{E} \times \mathrm{B}}$. For the $\mathrm{H}$-mode, there is a characteristic well in $\mathrm{E}_{\mathrm{T}}$ near the edge and a large gradient. The $\mathrm{E} \times \mathrm{B}$ velocity shear $\omega_{\mathrm{E} \times \mathrm{B}}$ is much greater then $\Delta \omega_{\mathrm{D}}$ near the edge, and gives rise to an ion transport barrier there. Performance of NCS discharges with internal transport barrier and peaked pressure profiles is limited by disruptions near the calculated beta limit. Calculations $[10,11]$ show that the stability limit can be increased by a broadening of the pressure profile. In DIII-D, the pressure profile can be broadened transiently by a controlled transition to H-mode during the NCS discharge. In the NCS discharge, it has been possible to extend the transport barrier and obtain ion heat transport in the neoclassical regime in the whole plasma.
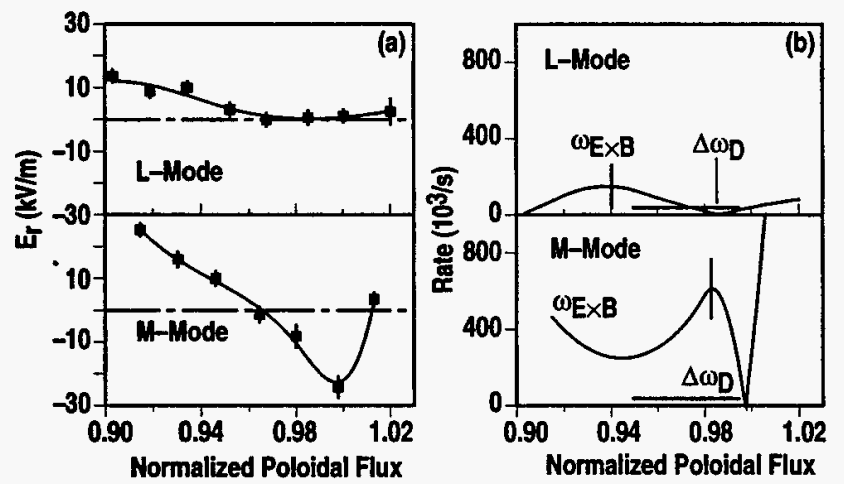

Fig. 2. Comparison of L-mode and H-mode edge profiles in DIII-D. In (a) the $\mathrm{E}_{\mathrm{r}}$ profiles are shown; notice the characteristic $\mathrm{E}_{\mathrm{r}}$ well at the plasma edge in $\mathrm{H}$-mode. In (b) the $\mathrm{E} \times \mathrm{B}$ shearing rate $\omega_{\mathrm{E} \times \mathrm{B}}$ is compared to the intrisic turbulence decorrelation rate $\Delta \omega_{\mathrm{D}}$. In $H$-mode the $E \times B$ shearing rate is greater than the turbulence decorrelation rate especially near the edge.

\section{B. Stability and Disruptions}

Wall stabilization enables high beta plasmas with broad current density profiles and broad pressure profiles. The effect on the plasma of a close wall is shown in Fig. 3. A factor two increase in normalized beta is calculated if the DIII-D wall is modeled as an ideal conductor, compared to the value without a wall. Of course, a real wall is resistive and will slow down the growth of the mode as opposed to stabilize it. However, plasma rotation is calculated to provide stability above the no wall limit. Experiments on DIII-D have confirmed resistive wall stabilization for many resistive wall times $[12,13]$. These experiments indicate that plasma rotation at the $q=2$ and 3 surfaces of only $1-2 \mathrm{kHz}$ is sufficient to maintain stability. Sustained plasma rotation and active non-axisymmetric feedback stabilization $[14,15]$ are presently thought to be able to overcome the resistive wall problem.

A disruption is the sudden rapid loss of the control of the plasma, which can lead to large forces on the vacuum vessel and internal components. In DIII-D, disruptions [16] can be divided into two basic classes, major disruptions and vertical displacement events (VDEs). In a major disruption, a MHD mode grows, leading to a loss of the thermal energy (thermal quench) and the resulting cold plasma suffers a rapid decay of current (current quench). In a VDE, the loss of vertical position precedes the thermal quench. As the plasma moves vertically toward the first wall, the cross section and the edge $q$ decrease until the shrinking plasma disrupts and loses thermal energy, typically when the edge $q$ approaches two.

There is a large variation in the current decay rate for a given current. However, the fastest decay rate for a full aperture plasma (volume $>19 \mathrm{~m}^{3}$ ) is $\mathrm{I}_{\mathrm{p} 0} /(\mathrm{dI} / \mathrm{dt}) \sim 4 \mathrm{~ms}$,

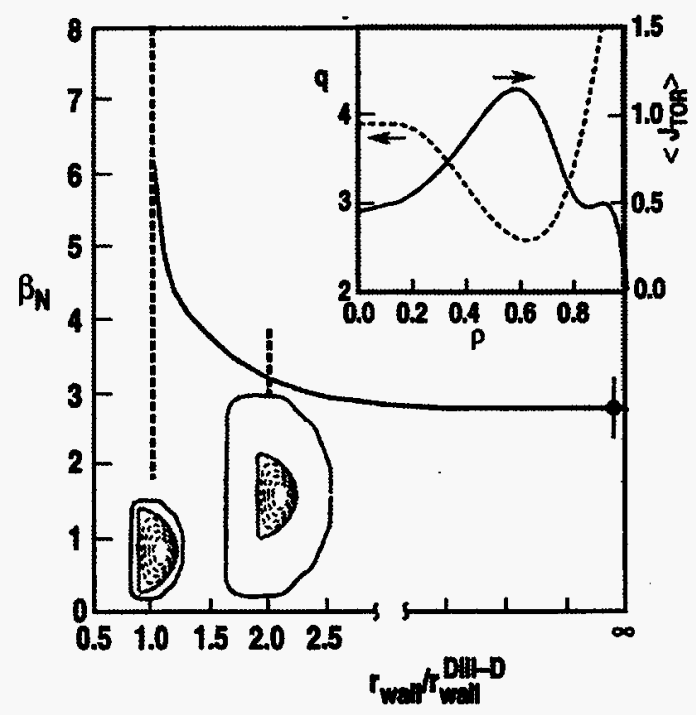

Fig. 3. Maximum stable beta increases for closer wall position. 
where $I_{p 0}$ is the pre-disruption current and $d I / d t$ is the average decay rate from $90 \%$ to $10 \%$ of the current. Discharges with small plasma aperture exhibit shorter decay time, approaching $2 \mathrm{~ms}$. The post-thermal quench equilibria for these discharges are vertically unstable and exhibit extremely rapid loss of vertical position. VDEs also have short decay rates, because the dual effects of reduced crosssection and reduced plasma temperature increase the plasma resistance.

A significant disruption related problem for future machines is the development of large poloidal "halo" currents that flow on the open field lines surrounding the plasma and return poloidally through the vessel. The interaction of these currents with the toroidal field causes large forces on the vessel and internal components. In order to better understand the driving mechanism for these currents, an experiment was performed in DIII-D in which the vertical feedback was turned off during the discharge. This triggers a VDE, and the poloidal and toroidal halo currents are measured with the current tile array (TCA) [17]. The time evolution of a VDE is shown in Fig. 4. Two mechanisms drive the poloidal halo current. After the thermal quench, the core plasma current starts to decay inducing toroidal current in the cold "halo" plasma on the open field lines. This current has a poloidal component given by $\mathrm{I}_{\text {halo }}(\mathrm{pol})=\mathrm{I}_{\mathrm{halo}}($ tor $) / q_{\text {edge }}$. A second mechanism for inducing poloidal halo current is the reduction of toroidal flux linked by the plasma as the cross section shrinks. In the early phase of the disruption, the two driving terms are about equal, but during the peak halo current the first mechanism dominates as shown in Fig. 4. This explains why the poloidal halo current is very small during the stage where the edge $q$ is very high. Poloidal halo currents of up to $40 \%$ of the pre-disruption current, $\mathrm{I}_{\mathrm{p} 0}$ have been measured
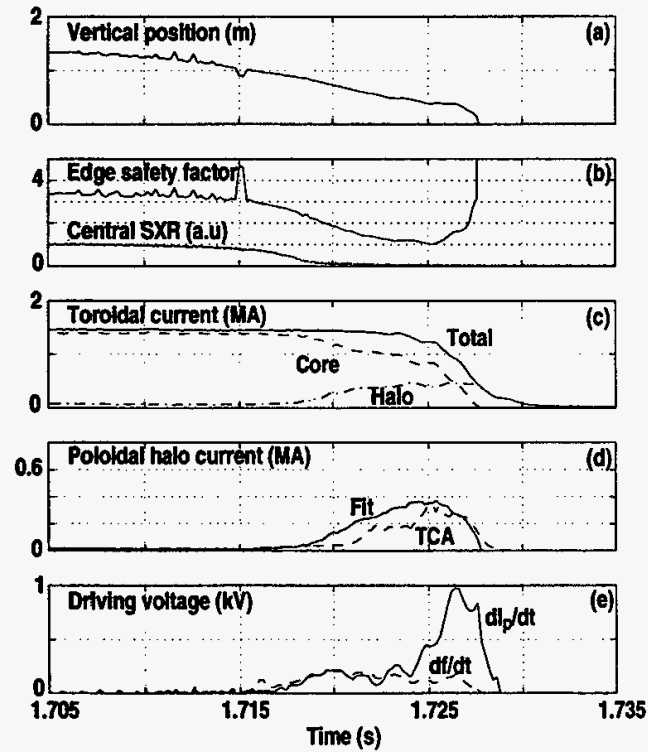

Fig. 4. Time evolution of a VDE. Poloidal halo current reaches $370 \mathrm{kA}$ during the decay of the toroidal plasma current. during VDEs. Toroidal asymmetries are observed in the poloidal halo current with peaking factors as high as 3 during the peak of the halo current. Normally these asymmetries rotate, but they can lock, thus increasing the effect of the asymmetry.

The combined effects of high halo currents, toroidal asymmetries and intense heat pulses during the disruption make mitigation of disruptions imperative. In DIII-D, we have explored the use of neon and argon pellet injection to mitigate disruptions and have shown that both are effective in significantly reducing the disruption effects. Fig. 5 shows a shot in which a $2.8 \mathrm{~mm}$ argon pellet is injected after the beginning of the vertical instability. The duration of the pellet ablation is $600 \mu \mathrm{s}$ and the current decay begins within $200 \mu \mathrm{s}$ of the end of the ablation. The loss of stored energy begins immediately with the pellet injection and is complete within $100-200 \mu \mathrm{s}$ of the end of the ablation. The internal induction of the plasma current profile increases during the ablation. The pellet causes growth of large $n=1$ and $n=2$ modes, which may play a role in the loss of the remaining thermal energy and the subsequent profile flattening. Both the $\mathrm{Ne}$ and $\mathrm{Ar}$ pellets significantly reduce the halo current magnitude and toroidal asymmetry. Typical reduction of the halo current was $30 \%-50 \%$ with the largest reductions for the highest halo currents. The disruption heat pulse to the divertor floor was reduced at least by $50 \%$. While pellet injection successfully mitigated the effect of the halo current and heat flux, it created run-away electrons. In a reactor, runaway electrons energies could exceed $25 \mathrm{MeV}$ and could damage the vacuum vessel.

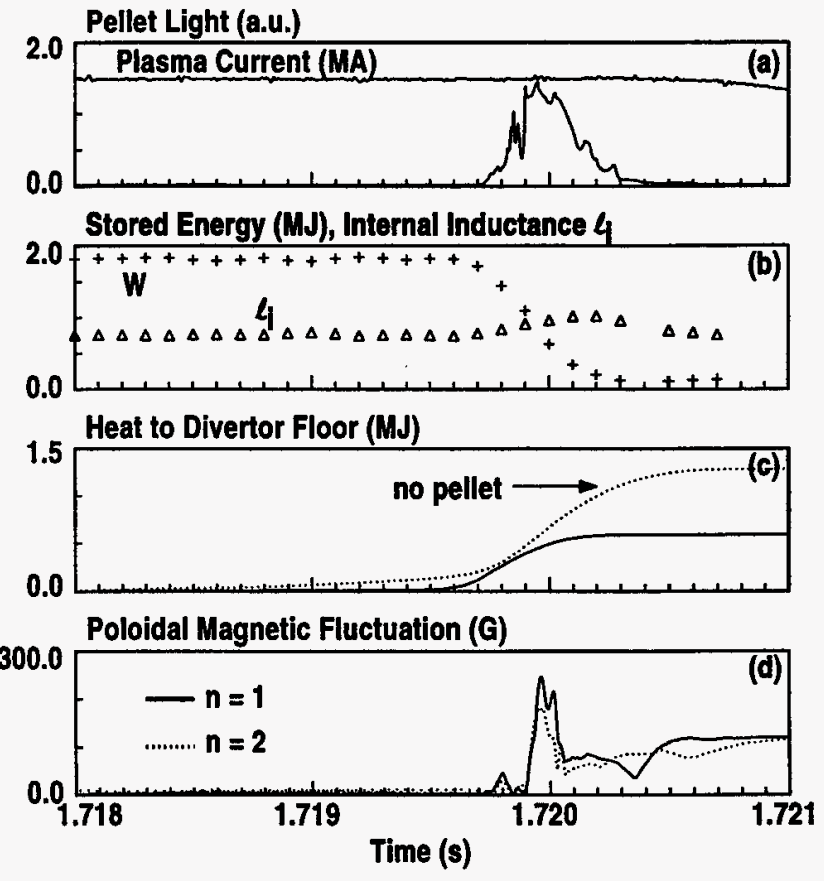

Fig. 5. Argon pellet injection into a VDE causes immediate loss of store energy and rapidly initiates the current quench. The heat conducted to the floor is reduced by $50 \%$ with injection of a pellet. 


\section{- C. Erosion Experiments}

The DiMES [18-20] probe is ta device whereby different samples can be inserted into floor of the DIII-D tokamak for exposure to the plasma edge and removed later for analysis. In order to validate the REDEP/WBC $[21,22]$ erosion code, a depth-marked graphite sample was inserted and exposed to 3-5 similar discharges with the outer strike point of the diverted plasma on the sample. The extensive set of divertor diagnostics provided measurements of the key plasma parameters (e.g. $n_{e}, T_{e}, \Gamma_{i}$ ) for input to the codes. REDEP predicts the features and magnitude of the measured net erosion: a net erosion region of $2-3 \mathrm{~cm}$ radial width centered slightly inboard of the separatrix with peak erosion rates ranging from $9 \mathrm{~cm} /$ burn-year (at $0.7 \mathrm{MW} / \mathrm{m}^{2}$ incident heat flux) to $45 \mathrm{~cm} /$ burn-year $\left(2 \mathrm{MW} / \mathrm{m}^{2}\right)$. The best agreement occurs when deuterium ion on carbon sputtering and selfsputtering yields for oblique incidence are used. Calculations excluding the effect of oblique incidence and the selfconsistent treatment of self-sputtering from redepositing particles underestimate the erosion by a factor of ten. Modeling shows that ELMs do not significantly enhance erosion in DIII-D due to their high density and recycling, low duty cycle and typical low energy densities $\left(15 \mathrm{~kJ} / \mathrm{m}^{2}\right)$, which fall below the threshold for carbon ablation. Long term exposure ( 9 months, 1400 lower single null discharges) of DII-D tiles [23] shows a local net erosion rate at the outer strike point of $30 \mathrm{~cm} /$ burn-year and an equivalent rate of redeposition at the inner strike point. This is in good agreement with the results of the dedicated DiMES exposures and REDEP modeling for typical outer strike point conditions on DIII-D. The redeposition patterns of Be and $W$ films on the graphite DiMES sample have been successfully reproduced by the WBC Monte-Carlo transport code. The results indicate that the near-surface transport of sputtered material (and hence redeposition efficiency) is determined mostly by ionization lengths and sheath acceleration. Experiments and modeling show that beryllium has a characteristic redeposition length 4-5 times that of tungsten due to tungsten's shorter ionization length.

\section{Divertors}

The ultimate goal of the DIII-D Divertor program [24] is to achieve a high performance core plasma which coexists with an advanced divertor plasma. The divertor plasma must reduce the heat flux at the divertor plate to acceptable levels; the current technique disperses the heat flux over a wide area by radiation. Two divertor pumps and baffles are installed in DIII-D as shown in Fig. 6. The lower divertor was installed first and results in an open divertor configuration, which pumps low triangularity plasmas. It can maintain the plasma density a factor 2 below the natural density in ELMing $\mathrm{H}$-mode [25] discharges. With $\mathrm{D}_{2}$ puffing and pumping, we have sustained radiative divertor operation (reduced heat load on the floor) with only slight degradation in con-

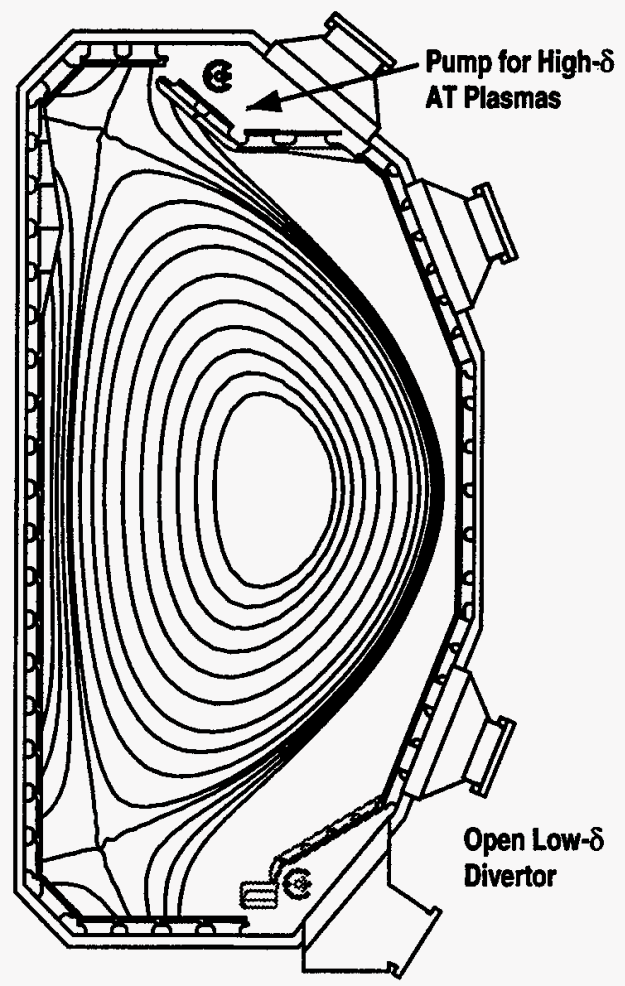

Fig. 6. The lower divertor is open and can pump low triangularity plasmas, whereas the upper divertor is more closed and can pump high triangularity plasmas.

finement [26], resulting in parameters close to those desired for ITER.

The new upper divertor baffle and pump was installed in the ceiling of DIII-D in early 1997. It is optimized for high triangularity plasma cross sections. Without plasma, the measured deuterium pumping speed was $\sim 40,000$ Torr $\ell / s$, similar to the lower pump. The slanted baffle is water-cooled to stabilize the plate temperature during a succession of plasma shots. Careful attention was paid to making the baffle region relatively gas tight. New magnetic pickup coils were mounted on the plate, and these are incorporated into the EFIT shape reconstruction. "Isoflux" shape control is used to position the plasma in the baffle and sweep the outer strike point close to the pumping aperture. An IR camera, which views the upper divertor, along with bolometer chords is used for tomographic reconstructions of radiated power from the divertor region. Two Langmuir probes near the entrance to the pump aperture are used to measure the ion flux into the pump and an ASDEX type gauge indicates the baffle pressure.

The experimental divertor data are use to validate computer models that are used to model ITER and other future divertor systems. The models have so far used the standard model, which is based on classical conduction of heat flow and constant pressure along the field lines, coronal equilibrium radiation rates, constant impurity concentration everywhere in the system, and sheath limited heat flow at the divertor 
flow. This model limits the total radiation to about $100 \mathrm{MW}$ maximum radiation in ITER. This suffices for the nominal power balance in ITER, but for advanced tokamak operations in ITER more radiation must be shifted from the core plasma into the divertor (to a maximum of $300 \mathrm{MW}$ in ITER). A new model supported by experimental data from DIII-D especially the divertor Thomson scattering system include impurity concentration in the divertor, non-coronal equilibrium radiation rates, convection flow of heat and fuel, non-Maxwellian enhancement of radiation, and plasma recombination (see Fig. 7). Initial data analysis from DIII-D indicated that the ITER advanced tokamak divertor requirements might be met by the new model.

\section{FUTURE PLANS}

Acceleration of increasing Electron Cyclotron Heating has high priority. With the successful testing of the two gyrotrons and development of new diamond windows, it appears that long-pulse gyrotrons are now available. The $\mathrm{ECH}$ power will be used for current profile control, perturbation studies, and transport barrier control. An extension of the DIII-D pulse length can be obtained by installing a new return bus for the toroidal coil, minor upgrades of the field shaping coil supplies. A longer tokamak pulse duration is important for stability studies; extension of the advanced tokamak mode and wall stabilization. Upgrade of the Thomson scattering system to include a central cord is important for transport and neutral density measurements, understanding of disruptions and to aid active mode control of neoclassical MHD modes. The top divertor will be completed with inner and private flux baffles, which should help reduce core fueling of neutrals and thereby improve confinement and increase the electron temperature for a more efficient current drive. Later, the lower divertor will be upgraded for advanced tokamak operations.

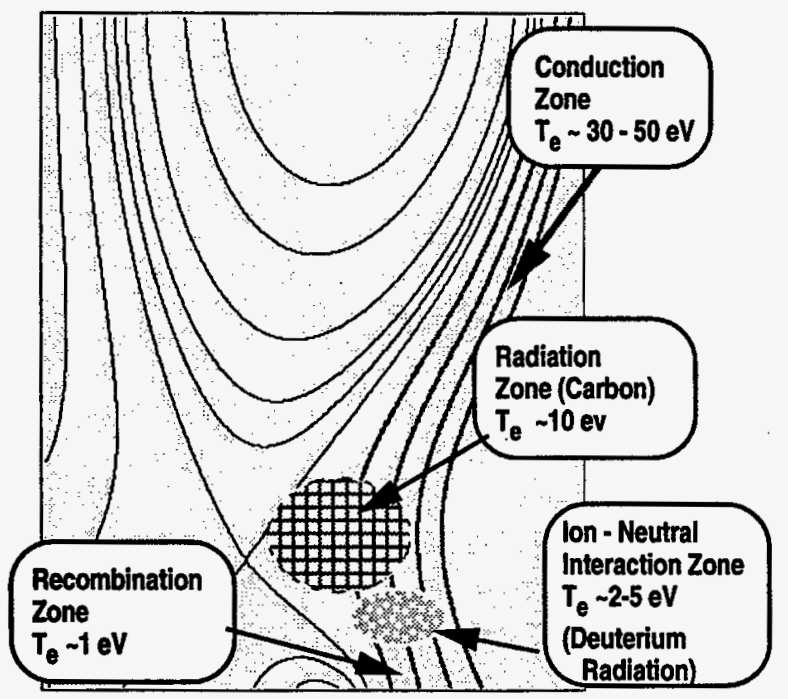

Fig. 7. Several processes determine the heat flow to the floor of the divertor. At $30-50 \mathrm{eV}$ the heat is conducted. At $10 \mathrm{eV}$ carbon radiation becomes important, at 2-5 eV the ions interacts with the neutrals, and finally at $1 \mathrm{eV}$ recombination takes place close to the divertor floor.

\section{AKNOWLEDGEMENTS}

It takes a large and diverse group of people to operate and maintain a tokamak and it diagnostics, and to plan the experiments, execute them, and publish the data. The effort of everyone including scientists, engineers, and operations personnel are greatly appreciated.

\section{REFERENCES}

[1] Walker M L et al. "Control of pPlasma poloidal shape and position in the DIII-D tokamak," this conference.

[2] B. B. Kadomtsev: "Tokamak Plasma: A Complex Physical System", Institute of Physics Publishing, 1992.

[3] T.S. Taylor, "Physics of Advanced Tokamaks," TwentyFourth European Conference on Controlled Fusion and Plasma Physics, June 9-14, 1997, Berchtesgaden, Germany.

[3] Goldstone R J 1984 "Energy confinement scaling in Tokamaks" Plasma Phys. Contr. Fusion 26 (1984)

[4] ASDEX team 1989 "The H-mode in ASDEX" Nuclear Fusion 29 (1989).

[5] C M Greenfield, et al., "The JET and the DIII-D teams, Plasma Phys. Controlled Fusion 35 (1993).

[6] E. J. Strait et al., 1995 Phys. Rev. Lett. 754421.

[7] K H Burrell at al. (1997), Effects of E x B velocity shear and magnetic shear in the formation of core transport barriers in the DIII-D tokamak.

[8] K H Burrell (1997) Effects of E x B velocity shear and magnetic shear on turbulence and transport in magnetic confinement devices. Phys. Plasmas 4.

[9] Turnbull A D, et al. 1996 Plasma Phys. And Contr. Nucl. Fusion Research 1996 Montreal (International Atomic Energy Agency, Vienna) to be published.

[10] Turnbull A D, et al., "Synergism Between Cross-Section and Profile Shaping in Beta Optimization of Tokamak Equilibria with Negative Central Shear" 1997 (to be published).

[11] Strait E J 1995, Phys Rev Lett. 742483 (1995).

[12] Taylor T S, et al. Phys. Plasmas 22390.

[13] Fitzpatrick R, 1996 Nucl. Fusion 361.

[14] Jensen T H and Fitzpatrick R, 1997 Phys. Plasmas 42997.

[15] Kellman A G et al. 1997 Plasma Phys. And Contr. Nucl. Fusion Research 1996 Montreal (International Atomic Energy Agency, Vienna) to be published.

[16] Evans, T E et al., " magnetic and thermal energy flow during disruptions in DIII-D" in Proc. 23rd Euro. Conf. On Contr. Fusion and Plasma Physics, Kiev, Ukraine, 1996 (European Physical Society, Petit-Lancy, Switzerland) to be published.

[17] Wampler W R, et al., J. Nucl. Mater. 233-237 (1996) 791.

[18] Whyte D G et al., "DiMES divertor erosion experiments on DIII-D", 12th Int. Conf. On Plasma-Surface Interactions in Controlled Fusion Devices, PSI-12, May 1996, St-Raphael, France, to be published in J Nucl Mater.

[19] Bastasz R et al., J. Nucl. Mater. 220-222 (1995) 310.

[20] Brooks J N, Phys. Fluids 8 (1990) 1858.

[21] Brooks, J N, Nucl. Technol./Fusion 4 (1983) 33.

[22] Hua T Q, J. Nucl. Mater. 220-222 (1995) 342.

[23] Allen S L et al. "Effects of divertor geometry and pumping on the plasma performance on DIII-D" Twenty-Fourth European 
Confernce on Controlled Fusion and Plasma Physics, June 9. 14, 1997, Berchtesgaden, Germany.
[24] Mahdavi M A, et al. J. Nucl. Mater. 220-222, 13 (1995).

[25] Petrie T W et al., Nucl. Fusion 37, 321 (1997).

S.L. Allen, ${ }^{(a)}$ P.M. Anderson, M.E. Austin, ${ }^{(b)}$ D. Baggest, F.W. Baity, ${ }^{(c)}$ D.R. Baker, D.E. Baldwin, L.R. Baylor ${ }^{(c)}$ G. Barber, ${ }^{(c)}$ C.B. Baxi, S. Bernabei, ${ }^{(d)}$ D. Bernat, J.A. Boedo, ${ }^{(e)}$ J.D. Broesch, N.H. Brooks, K.H. Burrell, R.W. Callis, G.L. Campbell, C. Campo, T.N. Carlstrom, E. Carolipio, $(f)$ B. Carreras, ${ }^{(c)}$ W.P. Cary, T.A. Casper, ${ }^{(a)}$ V.S. Chan, H-Y Chang, ${ }^{(g)}$ E. Chin, H. Chiu, S.C. Chiu, M.S. Chu, D.D. Clow, S. Coda, ${ }^{(h)}{ }^{\text {R.Colchin, }}{ }^{(c)}$ W. Davis, ${ }^{(d)}$ J.C. DeBoo, J.S. deGrassie, G. Diao, ${ }^{(i)}$ M. Di Martino, J.L. Doane, E.J. Doyle, ${ }^{(j)}$ T.E. Evans, M.E. Fenstermacher, ${ }^{(a)}$ J.R. Ferron, D.K. Finkenthal, ${ }^{(l)}$ R.K. Fisher, C.B. Forest, ${ }^{(m)}$ E. Fredrickson, ${ }^{(d)}$ J. Freeman, R.L. Freeman, A. Garofalo, ${ }^{(n)}$ T. Gianakon, ${ }^{(i)}$ P. Gohil, A.M. Gootgeld, D. Gray, ${ }^{(e)}$ K.L. Greene, C.M. Greenfield, R.J. Groebner, H.J. Grunloh, M.J. Hansink, T.E. Harris, R.W. Harvey, T. Hatae, ${ }^{(o)}$ W.W. Heidbrink, ${ }^{(f)}$ D.N. Hill, ${ }^{(a)}$ F.L. Hinton, T.R. Hodapp, D.J. Hoffman, ${ }^{(c)}$ J. Hogan, ${ }^{(c)}$ K.L. Holtrop, M.A. Hollerbach, R-M. Hong, W. Houlberg, ${ }^{(c)}$ C.L. Hsieh, D.A. Humphreys, A.W. Hyatt, H. Ikezi, R.C. Isler, ${ }^{(c)}$ G.L. Jackson, F. Jaeger, ${ }^{(c)}$ T.H. Jensen, T. Jernigan, ${ }^{(c)}$ R.W. Johnson, K.M. Keith, A.G. Kellman, D.H. Kellman, R. Khayrutdinov, ${ }^{(p)}$ K.W. Kim, ${ }^{(k)}$ Y. Kim, Y. Koide, ${ }^{(o)}$ C.C. Klepper, ${ }^{(c)}$ J.A. Konings, ${ }^{(q)}{ }^{\text {G. Labik, }}{ }^{(d)}$ R.J. La Haye, L.L. Lao, C.J. Lasnier, ${ }^{(a)}$ G.J. Laughon, E.A. Lazarus, ${ }^{(c)}$ B. Lee, ${ }^{(j)}$ J.-H. Lee, ${ }^{(j)}$ R. Lee, R. Lehmer, ${ }^{(e)}$ A.W. Leonard, J.A. Leuer, Y-R. Lin-Liu, J.M. Lohr, G. Lu, T.C. Luce, S. Luckhardt, ${ }^{(e)}$ V. Lukash, ${ }^{(n)}$ J.L. Luxon, M.A. Mahdavi, R. Maingi, $(c)$ C.C. Makariou, M.E. Mauel, ${ }^{(n)}$ W.B. McHarg, G. McKee, ${ }^{(m)}$ R. Meyer, ${ }^{(a)}$ K. Middaugh, R.L. Miller, P.K. Mioduszewski, ${ }^{(c)}$ C.P. Moeller, J. Moller, ${ }^{(a)}$ R.A. Moyer, ${ }^{(e)}$ M. Murakami, ${ }^{(c)}$ A. Nagy, ${ }^{(d)}$ G.A. Navratil, ${ }^{(n)}$ A. Nerem, D. Nilson, ${ }^{(a)}$ L.E. Nissley, R.J. O'Hara, R.C. O'Neill, P. O'Shea, ${ }^{(h)}$ T.H. Osborne, L. Owen, ${ }^{(c)}$ C.T. Parker, R. Patterson, W.A. Peebles, ${ }^{(j)}$ B. Penaflor, Q. Peng, P.l. Petersen, T.W. Petrie, C.C. Petty, D.A. Phelps, J. Phillips, D. Piglowski, R.1. Pinsker, P.A. Politzer, D. Ponce, G.D. Porter, ${ }^{(a)}$ R. Prater, S. Pronko, A. Reiman, ${ }^{(n)}$ E.E. Reis, D. Remsen, C. Ren, ${ }^{(m)}$ M.E. Rensink, ${ }^{(a)}$ C.L. Rettig, ${ }^{(j)}$ G. Rewoldt, ${ }^{(d)}$ T.L. Rhodes, ${ }^{(j)}$ B.W. Rice, ${ }^{(a)}$ J. Robinson, J. Rogers, ${ }^{(d)}$ M. Rosenbluth, ${ }^{(e)}$ E. Ruskov, $(f)$ S. Sabbagh, ${ }^{(n)}$ O. Sauter, ${ }^{(r)}$ J. Schachter, M.J. Schaffer, D.P. Schissel, J.T. Scoville, D.L. Sevier, T.C. Simonen, J.P. Smith, R.T. Snider, F. Söldner, ${ }^{(s)}$ G.M. Staebler, B.W. Stallard, $(a)$ R.D. Stambaugh, H. St. John, R.E. Stockdale, E.J. Strait, E. Synakowski, ${ }^{(d)}$ P.L. Taylor, T.S. Taylor, T. Terpstra, ${ }^{(d)}$ D.M. Thomas, S. Turgarinov, ${ }^{(p)}$ A.D. Turnbull, F. Waelbroeck, ${ }^{(b)}$ M.L. Walker, M.R. Wade, ${ }^{(c)}$ R.E. Waltz, J.G. Watkins, ${ }^{(t)}$ W.P. West, D.G. Whyte, ${ }^{(e)}$ D. Wròblewski, ${ }^{(u)}$ C.P.C. Wong, R.D. Wood, ${ }^{(a)} \mathrm{X}$. Wu, ${ }^{(v)}$ D. Zhang, ${ }^{(l)}$ and J. Zhang $(e)$

\section{PERMANENT ADDRESS}

(a) Lawrence Livermore Natl Lab., USA

(b) U. of Texas, Austin, USA

(c) Oak Ridge Natl Lab., USA

(d) Princeton Plasma Phys. Lab., USA

(e) U. of California at San Diego, USA

(f) U. of California at Irvine, USA

(g) KAIST,

(h) Massachusetts Institute of Technology, USA

(i) Southwestern Institute of Phys., China

(j) U. of California at Los Angeles, USA

(k) Fartech, (l) Palomar College, USA

(m) U. of Wisconsin, USA

(n) Columbia U., USA

(o) Japan Atomic Energy Research Institute, Japan.

(p) TRINITI, Russia

(q) FOM Inst., The Netherlands

(r) EPFL, Switzerland

(s) JET, United Kingdom

(t) Sandia Natl Labs, USA

(u) ORINCON Corp., USA

(v) ASIPP, China 


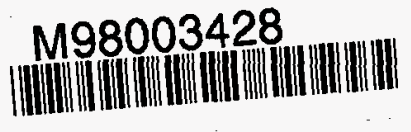

Report Number (14) GA--A22.748

Publ. Date (11) $\frac{199802}{E R, X F}$
Sponsor Code (18)
UC Category (19) 